\title{
Experimental On/Off Control of the Swing Phase of Paraplegic Gait Induced by Surface Electrical Stimulation
}

\author{
Henry M. Franken', Student Member, IEEE, Peter H. Veltink', Member, IEEE, Gert Baardman², \\ Ronald A. Redmeijer', Herman B.K. Boom', Member, IEEE \\ 1Biomedical Technological Institute, University of Twente, 7500 AE Enschede, The Netherlands \\ ${ }^{2}$ Roessingh R\&D, 7500 AH, Enschede, The Netherlands
}

\begin{abstract}
Parameterized swing phase of paraplegic gait was obtained by stimulating the main hip flexors, hamstrings, and quadriceps using surface electrodes. The hipflexors were stimulated to generate a desired hip angle range. The hamstrings provided footclearance in the forward swing. The quadriceps were stimulated to acquire knee extension at the end of the swing phase. Stimulation patterns were optimized on the basis of an experimental sensitivity analysis. During open-loop stimulation with the optimized stimulation patterns, the hip angle range varied in time due to the effects of potentiation and muscle fatigue. This time-dependent behaviour was succesfully compensated for using a cycle-to-cycle PID controller, which computed the hip flexor stimulation pattern on the basis of the achieved hip angle range in previous cycles.
\end{abstract}

\section{INTRODUCTION}

Functional Electrical Stimulation (FES) can restore or enhance motor functions, such as walking, in paraplegics [1].

Performing a task, as walking, for a prolonged time may be problematic because of fast fatigue of the artificially stimulated muscles [2]. For this reason, current FES-systems, having pre-programmed stimulation patterns which are applied in open-loop, are tuned such that, initially, exaggerated hip joint angles result, in order to provide a margin of safety at the time the subject's muscles become fatigued [3].

In the current paper, we parameterized the swing phase of paraplegic gait with desired objectives and derived stimulation patterns for the main swing-phase muscles to achieve these objectives. Parameterization of the swing phase facilitates adaption of the stimulation patterns from cycle-to-cycle. This cycle-to-cycle control concept was investigated by using a discrete-time PID controller [4]. The controller maintained the desired swing phase objectives by adjusting the stimulation patterns on the basis of the error between desired and actually obtained swing phase objectives in the previous swing cycles.

\section{METHODS}

\section{A. Optimization of stimulation patterns}

Swing phase objectives: When optimizing the stimulation patterns for the muscle groups of the consider system (Fig.
1), the motion of the leg was observed on the level of a swing cycle. The generated movement should satisfy the following desired objectives. The hip angle range should be approximately $45 \mathrm{deg}$, corresponding to natural human gait. The foot clearance in the forward swing should be sufficient ( $>1 \mathrm{~cm}$ ), in order to prevent the subject from stumbling. Knee extension was desired at the end of the forward swing to ensure safe body weight bearing during stance phase.

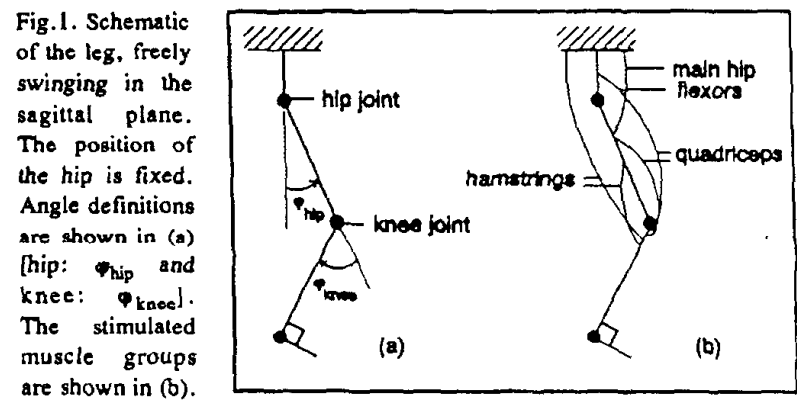

Stimulation timing: Each muscle was stimulated with one burst with constant stimulation frequency (hip flexors: $50 \mathrm{~Hz}$; quadriceps, hamstrings: $25 \mathrm{~Hz}$ ) at maximal recruitment each swing cycle. The burst times were minimized. Optimization of the timing was performed in three stages. First, the stimulation burst for the main hip flexors, yielding the desired hip angle range, was found. Subsequenty, the hamstrings were stimulated to obtain foot clearance. Finally, the quadriceps were stimulated to obtain knee extension. Cocontraction was accounted for by minor adjustments.

B. Cycle-to-cycle control strategy

A discrete-time PID controller, tuned on the natural frequency of the swinging leg, adjusted the burst time of the muscle groups to maintain the desired swing phase objectives. The obtained objectives in previous swing cycles were compared with the desired values and PID action was undertaken when deviation occured.

C. Subjects

Two complete T5-T6 level spinal cord injured patients participated. Both had been enrolled in an FES-training program of at least 6 months. Subject 2 exhibited signficant active spasm, limiting the funtional effect of the applied stimulation. 


\section{Experimental set-up}

Stand set-up: The subjects were positioned a controlled stand set-up with arm support. A self-fitting modular orthosis restricted the motion of the freely swinging leg in the sagittal plane. Hip and knee angle of the standing leg were fixed to zero. The standing leg was elevated by a block. Hip and knee angle of the swinging leg were measured with goniometers.

Electrode placement and data recording: Adhesive surface electrodes $(9 \times 5 \mathrm{~cm})$ were placed in the groin foil for hip flexor stimulation, over the quadriceps (rectus femoris, vastus lateralis/medialis) and over the hamstrings (near knee joint). The goniosignals were sampled at $100 \mathrm{~Hz}$.

\section{RESULTS}

Swing phase objectives: The optimized stimulation timing of the three muscle groups was similar in both subjects. It resulted in a satisfying swing (Fig. 2), although the achieved hip angle range was smaller in subject 2 (Subject 1: 45 deg; subject 2: $25 \mathrm{deg}$ ), due to active spasm and a less intensive training period.

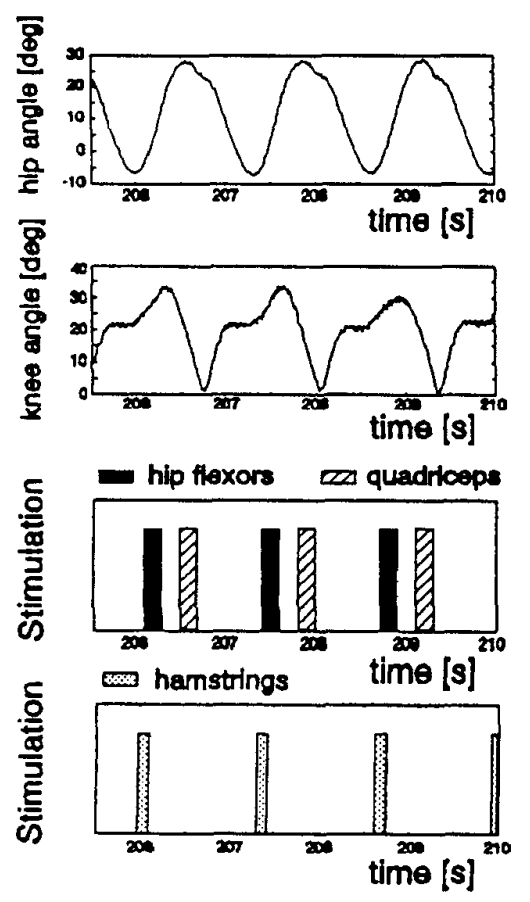

Fig. 2. Hip and knee angle registration with optimized stimulation timing for the msin hip flexors, hamstrings and quadriceps in subject 1 . The influence of each stimulation site can be detected in the hip and knee angle signal.

Open-loop control: During a prolonged period of openloop stimulation with the optimized stimulation patterns in subject 1, the hip angle range initially increased above the desired value due to the effect of potentiation in the hip flexors and subsequently decreased below the desired value due to muscle fatigue (Fig. 3a). The mechanical performance of the hamstrings, in maintaining sufficient foot clearance, and the quadriceps, in obtaining knee extension at the end of the forward swing, was relatively constant during the openloop trial.
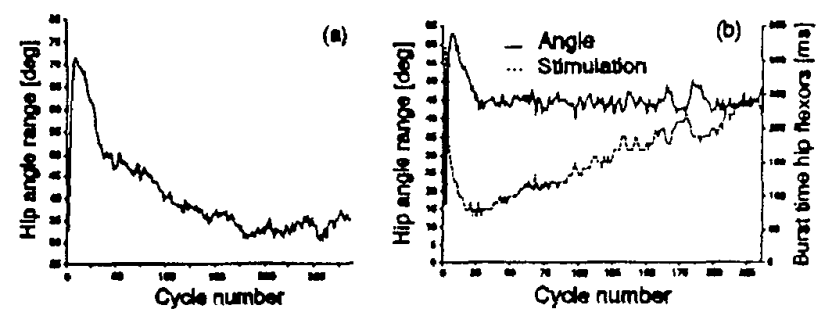

Fig. 3. Hip angle range per swing cycle. During the open-loop control trial (a) the three muscle groups were stimulated with the optimized stimulation pattems each cycle without adaption. During the cycle-to-cycle control trial (b) the burst time for the hipflexors was adapted. The quadriceps and hamstrings were stimulated in open-loop fashion with the optimized patterns.

Cycle-to-cycle control: It was observed during the open-loop experiments that only the hip flexor stimulation needed adaption. The PID controller was able to maintain a constant hip angle range by adapting the hip flexor burst time. Noteworthy, potentiation was adapted for by burst time decrement the burst time (initial part of Fig 3b). Muscle fatigue was compensated for by burst time increment.

\section{CONCLUSION AND DisCussion}

Our experimental results indicate that stimulation patterns can be derived such that swing phase objectives, characteristic for natural human gait, can be achieved in paraplegics.

Moreover, slowly time-varying system characteristics, such as the effects of muscle fatigue and potentiation, can be compensated for using a relatively simple control strategy, avoiding exaggerated hip joint angles succesfully. The desired movement can also be changed during locomotion by adapting the reference objectives for the controller.

Without orthosis, surface stimulation of the main hip flexors generated significant hip ad-and abduction during the forward swing, emphasizing the essential role of the orthosis.

\section{REFERENCES}

[1] B.J. Andrews, R.W. Barnett, G.F. Phillips and C.A. Kirkwond, 'Rulebased control of a hybrid FES orthosis for assisting paraplegic locomotion", Automedica, vol. 11, pp. 175-199, 1989.

[2] H.M. Franken, P.H. Veltink, M. Fidder, H.B.K. Boom, -Fatigue of intermittently stimulated paralyzed human quadriceps during imposed cyclical lower leg movements", Int. J. Elec. \& Kin., accepted, 1993

[3] E.B. Marsolais and R. Kobetic, "Functional electrical stimulation for walking in paraplegia ", J. Bone and Joint Surg., vol. 69-A, pp. 728-733, 1987.

[4] P.H. Veltink, "Control of FES-induced cyclical movements of the lower leg", Med. Biol. Eng. Comp., vol. 27, pp. NS8-NS12, 1991. 